\title{
REVIEW ON OPTICAL AND STRUCTURAL CHARACTERIZATION OF 2D TMDC SEMICONDUCTORS
}

\author{
Neelakandan.M.S ${ }^{1}$, Yadu Nath.V.K ${ }^{2}$ \\ ${ }^{1}$ International and Inter-University Center for Nanoscience and Nanotechnology, Mahatma Gandhi University, \\ Kottayam, Kerala, India \\ ${ }^{2}$ International and Inter-University Center for Nanoscience and Nanotechnology, Mahatma Gandhi University, \\ Kottayam, Kerala, India
}

\begin{abstract}
Nanoelectronics is an emerging field of technology, an interdisciplinary field where the principles of nanotechnology are applied in electronics. The present and emerging technology increasingly calls for compact, high yield, rapid and fairly effective devices for various applications. The emergence of nanoelectronics helps to make this kind of material for various applications. $2 D$ semiconductors are kinds of natural semiconductors having thickness of a very narrow range used to make Nanoelectronics devices such as transistors, sensors and etc. In this review, the research and advancements in the field of nanoelectronics and the influence of 2D semiconductors such as Graphene, transition metal dichalcogenides and black phosphorus in these developments are discussed. Also, the future applications and perspectives of these 2D semiconductors in nanoelectronics get discussed.
\end{abstract}

Keywords:- 2 D Semiconductors, Transition Metal Dichalcogenides, Photoluminescence, Second Harmonic Generation, Field Emission Transistors

\section{INTRODUCTION}

In order to raise the significance of nanoelectronics, it is beneficial to break the word into two components nano and electronics. The first word nano is referred to as the area of identifying objects which are very small in size, in the order of $10^{-9} \mathrm{M}$. Electronics may be synchronized as the technological and scientific branch dealing with electrical, or charged, components. Combined, the term Nano electronics refers to very small, electrically charged components. The name nanoelectronics cover selections of accessible fixtures and materials with common characteristics such as smaller interatomic interaction and quantum mechanical properties. The excruciating swallow whole crawl of structural circuits is at a gate length in nanoscale (50 $\mathrm{nm}$ and below) for transistors in CPUs or DRAM devices. Nanoelectronics have an important role in the field of computers, memory storage, optoelectronic devices and medical diagnostics.

In recent years 2D semiconductors such as graphene, transition metal dichalcogenides, and phosphorene have been found to have specific electrical and optical properties, which increased their importance in the fields of electronics, photonics, and optoelectronics. The research attention towards $2 \mathrm{D}$ semiconductors increased with the discovery of graphene in 2004 by Geim and Novoselov ${ }^{[1]}$. Compared to traditionally employed bulk materials, 2D monolayer semiconductor exhibits stronger piezoelectric coupling. The sensing and actuating applications with electronic devices can be aided by this characteristic property of 2D materials. Apart from graphene, monolayer TMDC (such as $\mathrm{MoS}_{2}$ and
$\mathrm{WS}_{2}$ ) and black phosphorus are considered as direct band gap semiconductors at monolayers, and have complementary properties to graphene ${ }^{[2-3]}$. 2D semiconductors have additional properties such as flexible mechanical properties, easy mode of fabrication and simple integration making them favorite materials for applications compared to traditional bulk materials ${ }^{[5-7]}$. Basically, all the requirements for the photonic applications can be fulfilled by these kinds of $2 \mathrm{D}$ semiconductors ${ }^{[8]}$. The combination of 2D material based electronic and photonic devices by implementing electronic properties to optical properties helps to achieve newly generated photonic-electronic circuits.

The 2D semiconducting materials have a variety of applications in the field of electronics and photonics. Transistors made of these materials are highly efficient in digital electronics. Free of dangling bonds at the interfaces produces some impure charges, which allow 2D semiconductors to work as a low-power instrument. The 2D semiconductor shows the ability to optimize and regulate thermal transfer leading to potential applications in nanocircuits. Another important application of these materials is in the field of light harvesting devices. Thin layer or monolayer of 2D materials can be suitable for fabricating flexible electronics and valley electronics ${ }^{[9-12]}$. This review will be discussing the optical and structural properties of 2D semiconductors (Transition Metal Dichalcogenides) and their application in the field of nanoelectronics. 


\section{2D SEMICONDUCTORS}

The emergence of new optical and electrical properties in the $2 \mathrm{D}$ semiconductors is due to quantum confinement, enhanced electron-electron interaction, and reduced symmetry and proximity effects. Here, properties of TMDC materials are being discussed through this review.

\subsection{Transition Metal Dichalcogenides}

In this review, a different kind of TMDC materials like $\mathrm{MoS}_{2}, \mathrm{MoSe}_{2}, \mathrm{WS}_{2}$, and $\mathrm{WSe}_{2}$ are being discussed, explaining its general properties such as electronic band structure, interaction with light (especially the luminescence properties, linear and nonlinear optical responses like SHG, mechanical and transport properties). These studies have very important role in the device applications for nanoelectronics. Transition metal dichalcogenides (TMDC's) are a type of 2D semiconductors with potential applications. These types of materials are composed of one transition metal atom placed in between two atoms from the chalcogenide group in a layered fashion. According to its potential applications, several kinds of TMDC materials are there. The review will be discussing the widely used TMDC materials like Molybdenum disulfide $\left(\mathrm{MoS}_{2}\right)$, Tungsten disulfide $\left(\mathrm{WS}_{2}\right)$, Molybdenum diselenide $\left(\mathrm{MoSe}_{2}\right)$ and Tungsten diselenide $\left(\mathrm{WSe}_{2}\right)$.

\subsection{Structure}

Transition metal dichalcogenides are a type of atomically thin layer semiconductor that have very important role in various fields. These are a particular kind of materials with a molecular formula of $\mathrm{MX}_{2}$. Here the $\mathrm{M}$ stands for the transition metal atom such as Molybdenum (Mo), Tungsten (W) or other transition metals and $\mathrm{X}$ is a chalcogenide atom like Sulfur S, Selenium Se and etc. The simple structure of dichalcogenides is a layer of Metal atom is placed between two layers of chalcogenide atom (Fig 1).

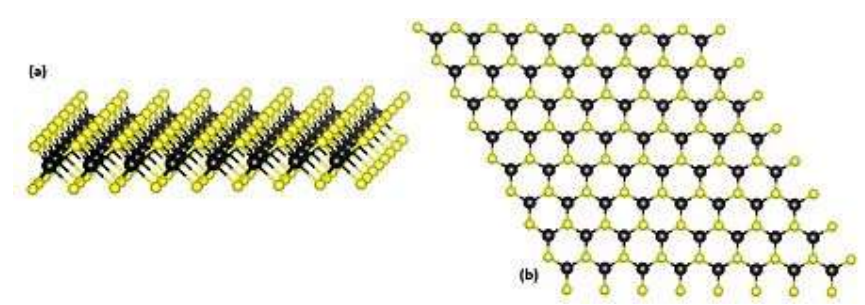

Fig 1: a) The structure of a hexagonally oriented TMDC monolayer. Black is metal atoms and yellow is $\mathrm{X}$ atoms. (b) Top view of TMDC monolayer

The thickness of a TMDC monolayer is in the order of Angstrom units. For example thickness of a monolayer of $\mathrm{MoS}_{2}$ is nearly $6.5 \AA$. As like in another 2D semiconductor graphene, the monolayers of TMDC are bound to each other through Van der Waal's force to form a bulk crystal. But the properties of the TMDC materials are entirely different from the properties of graphene. The important properties of TMDC which are entirely different from the graphene can be described as the optical and electrical properties. The band gap in the different monolayer TMDC materials like
$\mathrm{MoS}_{2}, \mathrm{MoSe}_{2}, \mathrm{WS}_{2}$, and $\mathrm{WSe}_{2}$ is a direct gap, where the conduction and valence bands have the constant momentum of electrons and holes in each energy states. This property helps to use TMDC materials in electronics applications as in the form of transistors and integrated chips and in optics as emitters and photo detectors ${ }^{[13]}$. Another interesting property is that the crystal structure of these kinds of materials has no inversion centers so that it permits to achieve a new degree of freedom carrier ${ }^{[14]}$. The research on TMDC monolayer is an emerging field of technology nowadays ${ }^{[15]}$. So in this review, the properties, applications and the future of TMDC monolayer materials get discussed in detail.

\subsection{Preparation of TMDC Monolayer}

There are different types of fabrication methods for the preparation of TMDC materials. Exfoliation, CVD, and molecular beam epitaxy are the widely used methods for the fabrication. Exfoliation is a simple method for the preparation of TMDC monolayer. It is a top-down approach, where the loosely bound layers can be produced by micromechanical cleavage ${ }^{[16]}$. TMDC crystals are made up of layers with weak Van der Waals force, which is clearly weaker, compared to chemical bonds. So the micromechanical cleavage like in graphene is used to produce TMDC monolayer. In this way, the TMDC monolayer can be produced by the Scotch tape mechanism, where the TMDC bulk crystal material is placed on the scotch tape and removed thereafter. Another piece of tape is placed on the TMDC layer on the tape and repeats the cleavage to get tiny flakes and place this onto the substrate. By peeling off the tape from the substrate surface, monolayer and multi-layers of TMDC are deposited on the substrate. Chemical Vapor Deposition, abbreviated as CVD is another method for the fabrication of TMDC monolayer, which is a kind of bottom-up approach where the reactants are delivered on the substrate and treated at $650^{\circ} \mathrm{C}$ in the presence of $\mathrm{N}_{2}{ }^{[17]}$. But the sample will be larger in size than obtained from the exfoliation technique. Molecular beam epitaxy is another good technique for the preparation of size-controlled monolayer of semiconducting material ${ }^{[18]}$. These are the widely used methods for the fabrication of TMDC monolayer. When comparing both these techniques, exfoliation is the simpler process for the preparation of the sample. But at the same time, CVD process can be used for producing the ultra-thin layers of TMDC materials with a better luminescent property.

\section{CLASSIFICATION OF TMDC MATERIALS}

\subsection{Molybdenum Disulfide}

This is classified as a TMDC material with a formula of $\mathrm{MoS}_{2}$, where Molybdenum is embedded in between two sulfur atoms in a sandwich form. These materials are relatively less reactive. In appearance and properties, $\mathrm{MoS}_{2}$ materials are similar to graphite. These materials have very low friction properties. In bulk nature, $\mathrm{MoS}_{2}$ has an indirect band gap with diamagnetic semiconducting nature similar to silicon with band gap energy of $1.23 \mathrm{eV}{ }^{[19]}$. Fabrication of $\mathrm{MoS}_{2}$ material is mainly done by the exfoliation methods. 
Chemical Vapor Deposition is used to make ultra-thin film coatings of $\mathrm{MoS}_{2}$. This exfoliated $\mathrm{MoS}_{2}$ will be twodimensional semiconductors of a thickness of few layers. The band structure of monolayer $\mathrm{MoS}_{2}$ is direct in nature. The direct band gap of $\mathrm{MoS}_{2}$ material has very wide applications in the field of optics, electronics, and optoelectronics. $2 \mathrm{H}$ and $3 \mathrm{R}$ are the two different polytypes existing in $\mathrm{MoS}_{2}$ with similar structure. In the case of $2 \mathrm{H}-$ $\mathrm{MoS}_{2}$, a trigonal prismatic coordination sphere is occupied by each Mo (IV) center and six sulfide ligands are bound to this sphere. Three Mo centers were connected to the sulfur center with a pyramidal configuration ${ }^{[20]}$. In this way, layered structure formed by the interconnection of trigonal prisms, where the molybdenum metal atoms are embedded in between the two sulfur layers. The layers of sulfur atoms in $\mathrm{MoS}_{2}$ monolayer have weak Van Der Waals force of attraction which leads to lubricant properties due to the low coefficient of friction ${ }^{[21]}$.

\subsection{Molybdenum Diselenide}

These materials are another kind of TMDCs with a formula $\mathrm{MoSe}_{2}$. IUPAC name for this material is that bis (selanylidene) molybdenum. The structure of $\mathrm{MoSe}_{2}$ is similar to the Molybdenum sulfide. Here transition metal Mo is sandwiched in between two selenium chalcogenide atoms causing a trigonal prismatic metal bonding coordination, leading to trilayers of Molybdenum diselenide ${ }^{[22]}$. Like other TMDC materials $\mathrm{MoSe}_{2}$ also has a layer structure with strong in-plane bonding interaction and low out of the plane interaction. Due to trigonal prismatic coordination, most common form of these TMDC materials is trilayers. When the material gets exfoliated the structure changes into octahedral. The metal ion in these compounds is surrounded by six Se2- ions. The coordination geometry of the Mo is sometimes found as octahedral and trigonal prismatic ${ }^{[23]}$. The bulk $\mathrm{MoSe}_{2}$ material can be prepared by reaction of molybdenum and selenium under high temperature and single crystal thick layers of $\mathrm{MoSe}_{2}$ fabricated by Chemical vapor deposition methods. $\mathrm{MoSe}_{2}$ monolayer can be prepared also by the micromechanical cleavage process. Comparing the mobility properties, the electron mobility of $\mathrm{MoSe}_{2}$ materials are significantly higher than that of the $\mathrm{MoS}_{2}$. In contrast to the graphene 2D, $\mathrm{MoSe}_{2}$ materials have direct band gap which will suggest applications in the field of transistors and detectors ${ }^{[24]}$.

\subsection{Wolfram Disulfide}

Wolfram disulfide or Tungsten sulfide is another kind of TMDC material with lot of potential applications. These kinds of materials naturally occur as rare minerals, as tungstenite with a formula $\mathrm{WS}_{2}$. As other TMDC materials $\mathrm{WS}_{2}$ also has a layered structure with $\mathrm{W}$ atoms situated in trigonal prismatic coordination sphere. Due to this layered structure of $\mathrm{WS}_{2}$ helps to fabricate inorganic nanotube, which was studied in earlier 90's ${ }^{[25]}$. The layered structure of bulk $\mathrm{WS}_{2}$ has a dark gray hexagonal crystals form. As in the case of $\mathrm{MoS}_{2}, \mathrm{WS}_{2}$ also shows the behavior of a dry lubricant. In the presence of oxygen atmosphere, $\mathrm{WS}_{2}$ converts to tungsten trioxide by heating. Thermalization in the absence of oxygen at $1250{ }^{\circ} \mathrm{C}$ leads to the decomposition of material into tungsten and sulfur ${ }^{[26]}$. Bulk wolfram sulfide can be synthesized in different ways such as thermolysis, in situ heating and etc. The monolayer of these materials can be produced by micromechanical exfoliation very easily.

\subsection{Wolfram Diselenide}

This is another type of TMDC material, which has very important role in solar cell applications and energy harvesting devices. Tungsten diselenide has a chemical formula of $\mathrm{WSe}_{2}$ with properties of an inorganic compound. Similar to molybdenum disulfide $\mathrm{WSe}_{2}$ also has a hexagonal crystalline structure. In this material tungsten, the atom is placed in between the two selenium atoms in a pyramidal geometry. In a manner of trigonal prismatic coordination sphere each of the tungsten atoms is covalently attached to six selenium ligands and each of the selenium atoms are attached to three of tungsten atoms in the geometry of pyramidal structure ${ }^{[27]}$. The bond length between the atoms of wolfram and selenium approximately equals $0.2526 \mathrm{~nm}$, and each selenium atoms is separated with a distance of $0.334 \mathrm{~nm}$. The force of attraction between the each monolayer in $\mathrm{WSe}_{2}$ is weak interactions. In the group-VI transition metal dichalcogenides, $\mathrm{WSe}_{2}$ is considered as the stable 2D semiconductor. Bulk wolfram sulfide is synthesized by the sputter deposition, i.e. heating thin tungsten films under high pressure from gaseous selenium with very high temperatures $(>800 \mathrm{~K})$ and the thin layers of $\mathrm{WSe}_{2}$ can be fabricated by the CVD process or micromechanical cleavage process ${ }^{[28]}$. These materials have very potential applications in the fields of solar cells and photovoltaic devices with LED properties and can be described in this review later.

\section{PROPERTIES OF TMDCS}

\subsection{Electrical Properties and Electronic Band}

\section{Structure}

$\mathrm{MoS}_{2}$ shows an electronic structure with a band gap that will be changed from indirect to direct from bulk to monolayer as the number of layers decreased ${ }^{[29]}$. In the bulk system $\mathrm{MoS}_{2}$ has an indirect band gap, i.e an interstate will be formed due to the different momentum of electrons in valence band and conductance band and momentum converted into the crystal lattice. But when the material gets into monolayer then the band gap changes into direct and the electrons have the same momentum in the valence and conductance band. Band structure studies show that the change in band gap from the bulk crystal to a monolayer of $\mathrm{MoS}_{2}$ material, the band gap becomes indirect to direct with an increase of band gap energy of $1.9 \mathrm{eV}$ from $1.2 \mathrm{eV}$. The band structure of TMDC monolayer materials was studied by DFT theories. Quantum confinement and change in hybridization in $\mathrm{P}_{\mathrm{z}}$ and d orbitals on $\mathrm{S}$ atoms and Mo atoms leads to the change in band structure with layer number. The Atomic structure has a strong correlation with the electronic structure of the material. Near the $\mathrm{K}$ point, direct excitonic states do not depend upon the change in the layer, but the $\Gamma$ - 
point shifts drastically from lower to larger in a manner of indirect one to direct one. In $\mathrm{MoS}_{2}$ the band gap for the bulk material is nearly $1.2 \mathrm{eV}$ and $1.8 \mathrm{eV}$ for one monolayer it is near. Change in layer thickness changes the energy of direct excitonic transition at the Brillouin zone but the indirect band gap increases with the decrease in layer number. Monolayer $\mathrm{MoS}_{2}$ have very high indirect transition energy when the material transforms into a 2D semiconductor with direct band gap. The photoluminescence spectrum becomes stronger with the enlargement of the indirect band gap in thinner $\mathrm{MoS}_{2}$.

As like the other TMDC materials electronic structure of $\mathrm{MoSe}_{2}$ molecules varies according to the change in thickness. As the thickness of material reduced, the material should change its band gap from indirect to direct. In all TMDC materials, the quantum confinement and spin interaction between the molecules are the important reason for the change in the band gap. The hybridization of Mo in $4 d$ orbital and $\mathrm{Se}$ at $4 \mathrm{p}$ states leads to the formation of the valence and conduction band in $\mathrm{MoSe}_{2}{ }^{[30]}$. From $\mathrm{MoS}_{2}$ to $\mathrm{MoSe}_{2}$ the both direct and indirect band gaps eventually decreases. Due to these reasons, $\mathrm{MoSe}_{2}$ shows a direct band gap in monolayer ${ }^{[31]}$.

Electronic structure of $\mathrm{WS}_{2}$ is very interesting like other TMDCs. In the bulk crystal, it shows an indirect band gap and then changes into direct with a decrease in the layer thickness. The electronic band structure of $\mathrm{WS}_{2}$ is like as $\mathrm{MoS}_{2}$. The bandgap of the materials increases gradually with decreasing the thickness of the material ${ }^{[32]}$.

\subsection{Photoluminescence Properties}

Luminescence properties of TMDC materials are the very important tool to study the change in band gap through indirect to direct by the decrease in thickness of the material. Strong photoluminescence emission is formed due to the transition from bulk material to the monolayer, which results in the formation of direct bandgap from indirect band gap.

Photoluminescence of $\mathrm{MoS}_{2}$, significantly, increases with decreasing layer thickness, and that strongest peak of luminescence produced from a monolayer while there is no spectrum in the bulk material. This unusual luminescence behavior is also predicted by some recent theoretical works; $\mathrm{MoS}_{2}$ shows an indirect band gap in bulk form gets converts into a direct band gap semiconductor when exfoliated to the monolayer. The experimental results and theoretical prediction about band gap strongly agree with each other. Figure-2 illustrates the photoluminescence spectrum of a monolayer TMDC material. This photoluminescence emission in the monolayer is absence in bulk $\mathrm{MoS}_{2}$ because of its indirect band gap like silicon. In bulk $\mathrm{MoS}_{2}$, no photoluminescence is observable. That is, compared to the incident electric field, the local electric field of a high refractive index material like $\mathrm{MoS}_{2}$ is much weaker. For ultrathin $\mathrm{MoS}_{2}$ layers, the local electrical fields are weaker, so the photoluminescence intensities and number of layer dependence was inversely proportional to each other. This unusual property of photoluminescence points out that luminescence quantum efficiency is higher in monolayer $\mathrm{MoS}_{2}$ compared to the bulk ${ }^{[33]}$. The d-orbital interactions in the $\mathrm{MoS}_{2}$ material cause the unusual PL difference with a decrease in layer number may also see in other transition metal dichalcogenides like $\mathrm{WS}_{2}, \mathrm{WSe}_{2}$, and $\mathrm{MoSe}_{2}$. This leads to a new technique, rich d-electron physics for controlling electronic structure in nanoscale materials ${ }^{[34]}$.

Bulk $\mathrm{MoSe}_{2}$ shows the energy of oblique band gap in between the near-infrared region at $1.1 \mathrm{eV}(1.13 \mu \mathrm{m})$. The direct excitons are better in power at $1.57 \mathrm{eV}(790 \mathrm{~nm})$ and $1.82 \mathrm{eV}(682 \mathrm{~nm})$ respectively. From fig-2 it shows the photoluminescence spectrum of exfoliated $\mathrm{MoSe}_{2}$. The monolayer $\mathrm{MoSe}_{2}$ indicates prominent peak at $1.57 \mathrm{eV}$ (792 $\mathrm{nm})^{[35]}$. Each emission spectral line is asymmetric in nature with a pointy edge in higher energy and flat tail at lower energy. compared to the bulk substances, PL intensities for monolayer substances are 10 - 20 instances stronger results in wide maxima at 1.53 and $1.35 \mathrm{eV}(812$ and $922 \mathrm{~nm})$. This shows that the indirect band increases in energy, while at the $\mathrm{K}$ point; direct gap has a constant energy when decreasing the thickness from bulk to monolayer materials ${ }^{[36]}$. Because of the strongly localized d orbitals at metal atom points, most of the valence band and minimal of conduction band originates on the K-point (direct gap). Therefore, the direct band gap decreases with lowering the number of layers. Corresponding to the measurements of $\mathrm{MoS}_{2}$, PL intensity of $\mathrm{MoSe}_{2}$ located in a decreasing manner with one order of value consistent with an extra layer ${ }^{[37]}$. For a $\mathrm{MoSe}_{2}$ trilayer there will be two properly-separated maxima; with one among them having the same strength as for the bilayer and the opposite assigned to the oblique band gap.

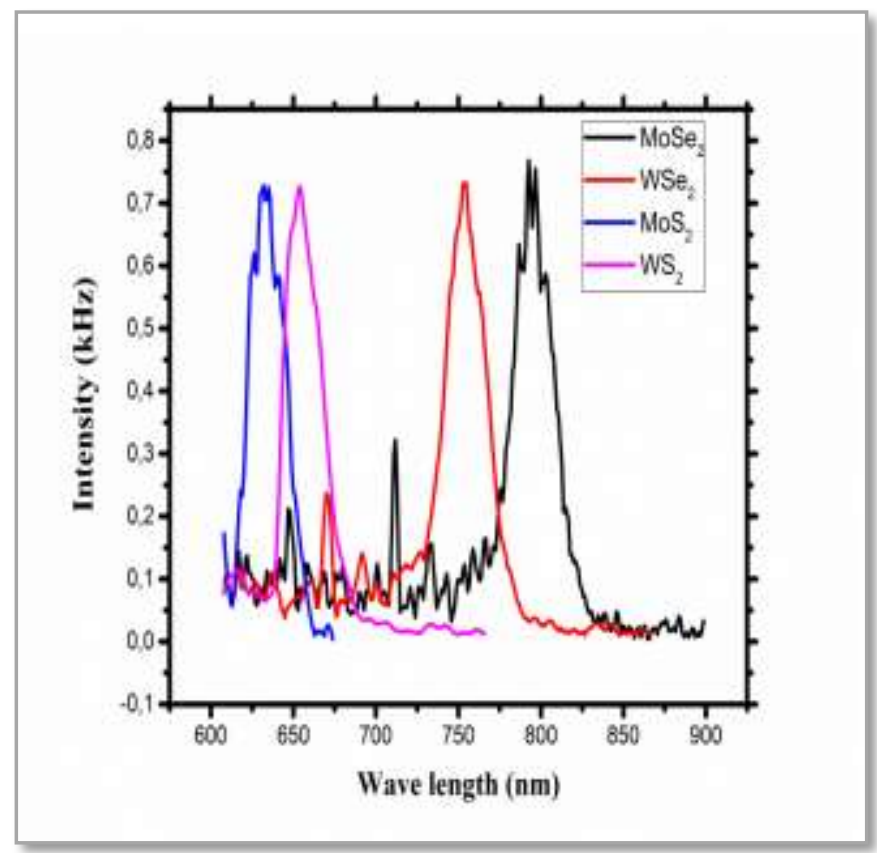

Fig-2 PL intensities from different TMDC monolayers

As shown in the previous case of $\mathrm{MoS}_{2}$ bulk, $\mathrm{WSe}_{2}$ wellknown shows an indirect band gap in the close to infrared at $1.2 \mathrm{eV}(1.03 \mu \mathrm{m})$. The direct excitons are shown in between the range $1.4-1.8 \mathrm{eV}(886-689 \mathrm{~nm})$ and at $2.30 \mathrm{eV}(540$ 
$\mathrm{nm}$ ), respectively ${ }^{[38]}$. Unique photoluminescence of monolayer $\mathrm{WSe}_{2}$ centered at $1.65 \mathrm{eV}(752 \mathrm{~nm})$ can be seen from fig-2. Now not the best PL spectrum but additional emission spectrum shows the asymmetry for $\mathrm{MoSe}_{2}$. Bilayer $\mathrm{WSe}_{2}$ emits at $1.54 \mathrm{eV}(806 \mathrm{~nm})$ with a side most of $1.60 \mathrm{eV}$ (773 nm). An interesting component in the PL depth of $\mathrm{WSe}_{2}$ bilayer is that the intensity most effectively decreased via a factor of 4 as compared to the monolayer. For a trilayer of $\mathrm{WSe}_{2}$, as in the case of the monolayer, the facet maximum has the equal position. Previous research on $\mathrm{MoS}_{2}$ suggests a robust relation with this as the stronger maximum on the lowest energy side is assigned to the indirect band gap. Differences in the relative intensities of the emission maxima might be related to the distinct excitation wavelength of $514 \mathrm{~nm}$.

For $\mathrm{WS}_{2}$ materials, as like in different TMDC substances luminescence characteristics of those kinds of substances additionally, have an important relation between the layer numbers. On the $\mathrm{K}$ point, direct gap transitions lead to the excitonic absorption peaks $\mathrm{A}$ and $\mathrm{B}$, respectively, around 625 and $550 \mathrm{~nm}$ for $\mathrm{WS}_{2}$. In $\mathrm{WS}_{2}$ there's also another peak is formed due to the optical transitions of the density of states peaks between the valence and conduction bands. Figure-2 also suggests the PL spectrum of different layers of $\mathrm{WS}_{2}$ materials and the PL dependence on the number of layers ${ }^{[39]}$. From monolayer $\mathrm{WS}_{2}$ it is seen that strongly more suitable $\mathrm{PL}$ focused at $630 \mathrm{~nm}$ due to direct gap excitonic recombination. As compared to the bulk form, the emission is 100 to 1000 time's stronger in monolayers. Monolayer $\mathrm{MoS}_{2}, \mathrm{MoSe}_{2}, \mathrm{WS}_{2}$ and $\mathrm{WSe}_{2}$ with a direct band gap in the visible and NIR frequency ranges, respectively, are novel building blocks for realizing unique heterostructures with tailor-made optoelectronic, electro catalytic, and photocatalytic functionalities.

\subsection{Optical Properties}

In the fields of electronics, spintronics and valley electronics, optical properties of TMDC materials have fundamentally very important applications. Photoluminescence characterization is the important tool for getting the important information associated with the optical band structure of the materials. Spectroscopic ellipsometry is used to study the optical characterization of the materials deeply. Spectroscopic ellipsometric measurements allow the materials optical functions such as refractive index of the material and coefficient of extinction. The magnitude of band gap and exciton binding energy in monolayer TMDCs has a direct correlation between the optical functions. The studies on the optical properties of TMDC materials will enhance the advancement in the field of existing optoelectronics and spintronic ${ }^{[40]}$.From the studies on optical functions, the refractive index of the TMDC monolayer materials increased with increase in wavelength for a wavelength ranging from $193 \mathrm{~nm}$ to $550 \mathrm{~nm}$ and approaches maxima then it decreased gradually until 1700nm. Two items merit special attention. First, the refractive index to a relentless worth of 3.5-4 within the NIR frequency vary with many abnormal dispersion options below 800nm.
The lack of inversion center or center of symmetry in the crystal lattice of monolayer TMDC materials leads to nonlinear optical characteristics like Second Harmonic Generation (SHG). SHG is used for the studies on the thickness of the materials and to find the different crystal structures ${ }^{[41]}$. The thickness dependence on the SHG is irregular. Second harmonic intensities are presented as functions of dichalcogenides and silicon oxide layer thicknesses. The second harmonic generation (SHG) microscopy is a very useful and powerful modality to study the local symmetry of the TMDC nanolayers and flakes. SHG studies are used to study the local symmetry of the dichalcogenides materials. Second-harmonic generation is a dipole allowed only in the non-centrosymmetric system. The symmetric criterion for SHG is:

$$
\mathbf{P}_{\mathrm{i}}=\chi^{(2)}{ }_{\mathrm{ijk}} \mathbf{E}_{\mathrm{j}} \mathbf{E}_{\mathrm{k}}
$$

In the case of symmetry of $\mathrm{MoS}_{2}$, SHG is used to find the polytypes of $\mathrm{MoS}_{2}$ materials with a thickness in the range of 3-200nm. With the use of GaAs wafer, the nonlinear susceptibility can be estimated. Studies done by E.D.Mishina et al. on the SHG of $\mathrm{MoS}_{2}$ shows that there are two different regions $\mathrm{A}$ and $\mathrm{B}$, with thicknesses of $10 \mathrm{~nm}$ and $50 \mathrm{~nm}$ possess quite homogeneous and nonhomogeneous behavior in the SHG intensity respectively.

A high SHG intensity was found not only for a countable number of atomic layers but also for thick flakes of up to $150 \mathrm{~nm}$. Although all flakes were single crystals, the brightness will vary with varying the parts of them in the SHG images. This kind of non-homogeneity in materials shows the property of polytypism, i.e. this confirms the presence of centrosymmetric $2 \mathrm{H}$ and non-centrosymmetric $3 \mathrm{R}$ polytypes in a source bulk sample, which is made into the flakes. SHG intensity dependences on the Azimuthal position and on the flake thickness and the PL spectra are used to analyze this property ${ }^{[42]}$. This kind of polytypism in TMDC Materials will be very helpful in device fabrication techniques.

In the case of other TMDC materials such as $\mathrm{WS}_{2}$, the SHG properties are very interesting. In the SHG images, all the edges of layered materials are seen as dark bands. In this case, the dark bandwidth and the resolution limit are coinciding at the point of FWHM approximately at $0.5 \mu \mathrm{m}$. Increase in the SH signal intensity at layer edges are often related to the very fact that the concentration of halogen molecules mixed into the Van der Waals gap within the regions adjacent to small crystal boundaries is way above the worth averaged over the microcrystal surface ${ }^{[43]}$.

From these studies, $\mathrm{WS}_{2}$ crystals are crystallized in the form of the $2 \mathrm{H}$ polytypes (i.e., they are centrosymmetric). All $\mathrm{WS}_{2}$ crystal layers are centrosymmetric, and all dark edges are EFISH manifestations. SHG effect in these kinds of materials can be mainly formed due to two effects. The first impact is owing to the presence of halogen molecules getting into the Van der Waals gap between the monolayers. The second effect is due to cause by band bending at microcrystallite edges ${ }^{[44]}$. This kind of different NLO properties leads to the device fabrication application by the use of TMDC materials. 


\subsection{Mechanical Properties}

Compared to other 2D materials like graphene, TMDC material can be strained up to $25 \%$. The applied strain has a linear proportion with the band gap of material. When the strain is applied to the material there is a linear decrease in the band gap of the material. The important thing, in this case, is that the indirect band gap increases with an applied strain to the monolayer than the direct band gap. As a result, the emission efficiency of a highly strained monolayer sample becomes very low. This property aids in the making of some flexible electrical devices with the use of strained TMDC materials. Recently, tuning of the band gap of TMDC material has become easier by the help of strain engineering, which does not alter the properties of single layers. Applying both tensile and shear strain (expansion and compression applied in different directions) to the monolayers in the parallel direction of its basal plane and computing its electronic band formation by DFT theories helps to study the mechanical effect of TMDC materials such as $\mathrm{MoS}_{2}, \mathrm{WS}_{2}, \mathrm{WSe}_{2}$ and $\mathrm{MoSe}_{2}{ }^{[45]}$.

Dependence of strain on electronic properties of TMDC semiconducting materials ( $\mathrm{MX} 2)(\mathrm{M}=\mathrm{Mo}, \mathrm{W}$ etc. and $\mathrm{X}=$ $\mathrm{S}$, Se) studied by the optimized geometry of monolayer was found by the relaxation of both atomic positions and lattice vectors. Tensile and shear strain apply to TMDC materials through various approaches for studying the mechanical strain effect on the electrical characteristics of semiconducting TMDCs. There are three different ways for applying the tensile strain, such as uniaxial growth of monolayer in $\mathrm{x}$-direction (xx), y-direction (yy), and homogeneous biaxial growth in each $\mathrm{x}$ and $\mathrm{y}$-directions $(\mathrm{xx}+$ $y y)$. Whereas increasing and decreasing the monolayer in $\mathrm{x}-$ and $y$-directions (xx yy) and by pressing and increasing in $\mathrm{x}$ direction and y-direction (yy $\mathrm{xx}$ ) at the same time with constant magnitude of strain are two ways for applying pure shear strain.

Application of strain on the lighter material $\mathrm{MS}_{2}(\mathrm{M}=\mathrm{Mo}$ or $\mathrm{W})$ shows that the shortest transition for an unstrained system otherwise will be a direct band transition, transformation to an oblique band transition which is related to (i) while the monolayer is stretched inside the course of $\mathrm{x}$ it's far $\Gamma-\mathrm{K}$; (ii), whilst it's miles stretched in y-direction $\Gamma_{-} \mathrm{L}$; and (iii) when the sheet is homogeneously stretched in each $x^{-}$and $y$-guidelines it's far $\Gamma_{-} \mathrm{K}$ and $\Gamma_{-} \mathrm{L}$, an examination of the band structure of strained $\mathrm{MoS}_{2}$ indicates that influence of tensile strain $(x x, y y, x x+y y)$ outcomes valence band shift in a downward path around $\mathrm{K}$ and $\mathrm{L}$ factors, but does not affect the $\Gamma$ point. The PDOS (Partial density Of States) and band shape were analyzed for reading the band transition of unstrained structures.

In the case of heavier dichalcogenides atoms $\mathrm{MSe}_{2}(\mathrm{M}=\mathrm{Mo}$, $\mathrm{W}$ ) it is just like $\mathrm{MS}_{2}$ type substances where the band gap decreases rapidly while $\mathrm{MSe}_{2}$ increased concurrently in each $\mathrm{x}$ - and y-instructions. For $\mathrm{MSe}_{2}$, larger pressure is required to acquire semiconductor to metal transition. The characteristics of heavier TMDC materials show different behavior compared to lighter ones upon the application of pure shear strain. Compared to the other case of natural shear strain (xx-yy), for $\mathrm{MSe}_{2}$ substances, the band gap decreases hastily when the system is stretched toward the $y$ direction and compressed thru $\mathrm{x}$-direction (yy-xx). Application of mechanical strain onto the semiconducting TMDC's helps to tune and control the electronic structure. So these materials can be used as a potential alternative to graphene and used for fabricating a variety of tunable nanodevices.

\subsection{Transport Properties}

Scattering and transport properties of 2D TMDC layers are bound to the plane of the material. The carrier of mobility of materials mainly influenced by the following principle disseminating instruments such as coulomb scattering, phonon scattering surface phonon scattering and etc. ${ }^{[46]}$. Layer thickness, temperature effects, the mass of carrier, carrier density electronic band structure and carrier thickness also influence on the transport properties. The carrier mobility of a material will increase with the increase in temperature. Partial ionic bonds in TMDCs increase the mobility of carriers as a relation between temperature and mobility. The optical phonon parts dominate at the higher temperature $^{[47]}$.

The mobility at room temperature is constrained to $\sim 410$ $\mathrm{cm}^{2} \mathrm{~V}-1 \mathrm{~s}-1$, essentially inferable from optical phonons ${ }^{[48]}$. Coulomb scattering in 2D TMDCs is brought about by arbitrarily charged debasements situated inside the monolayers of TMDCs or on its surface. Mobility of the material can be improved by providing suitable dielectric environments for $\mathrm{MoS}_{2}$. This Coulomb impact additionally, by and large, restrains the mobility of monolayer graphene around $10000 \mathrm{~cm}^{2} \mathrm{~V}^{-1} \mathrm{~s}^{-1}$ when it is set on gently dielectric materials, for example, $\mathrm{SiO}_{2}$. With the use of impurities, the concentration of carrier and band gap can be altered. The impact of concentration of carrier with temperature on the mobility of $\mathrm{MoS}_{2}$ is also decreases exponentionally ${ }^{[49]}$. Furthermore, unattached $\mathrm{MoS}_{2}$ has been appeared to have similar swells to those in graphene, which may likewise add to scattering and decrease in mobility. The calculated mobility of $\mathrm{MoS}_{2}$ at room temperature by the restriction of phonon scattering is $\sim 410 \mathrm{~cm}^{2} \mathrm{~V}^{-1} \mathrm{~s}^{-1}$, and comparable qualities are normal for other single-layer TMDCs.

Electrical conductivity measurements for $\mathrm{MoSe}_{2}$ monolayer is carried out by the use of DC probe techniques. From these measurements, it is seen that the electrical conductivity of $\mathrm{MoSe}_{2}$ monolayer was approximately in the order of 10-4 $\Omega^{-}$ ${ }^{1} \mathrm{~cm}^{-1}$ at room temperature. Compared to other materials, electrical conductivity values for $\mathrm{MoSe}_{2}$ is very lesser due to several reasons such as nano-crystallinity of film, presence of surface states and less thickness of the film. Electrical conductivity of this material increases as in the case of a semiconductor, where conductivity increases exponentially with elevation in temperature exponentially. From the Arrhenius law for thermally activated conduction $\left(\sigma=\sigma_{0} \mathrm{e}^{(-}\right.$ $\Delta \mathrm{Ea} / \mathrm{kBT}$ ), where $\Delta \mathrm{Ea}$ is the activation energy), i.e., a linear curve with a slope of $-\mathrm{Ea} / \mathrm{kB}$ will result when plotting $\ln \mathrm{s}$ with $1 / T^{[50]}$. The obtained activation energy from this slope 
was $0.61 \mathrm{eV}$. These kinds of highly modified transport properties of TMDC materials make it a good thing for the flexible transistor material.

\section{APPLICATIONS OF TMDC MATERIALS}

Some potential features of Semiconducting 2D TMDCs make them an attractive material for the application in FETs. The absence of unsatisfied valence, stable structure, and good transport properties compared to $\mathrm{Si}$ are the important properties of these materials. In 2004 the first FET was prepared with the use of $\mathrm{WSe}_{2}$ crystals with a mobility equivalent to Si FETs (P-type conductivity up to $500 \mathrm{~cm}^{2} \mathrm{~V}^{-}$ ${ }^{1} \mathrm{~s}^{-1}$ at room temperature). This material is bipolar in nature and proportion of on/off ratio is 104 at a temperature of 60 $\mathrm{K}{ }^{[51]}$.The first experiment in the field of a top-gated transistor with monolayer $\mathrm{MoS}_{2}$ was accounted for by Kis and coworkers. He made the device with a brilliant on/off ratio in the order of $\sim 10^{8}$. Compared to FET this device shows an n-type conduction, with a mobility of $>200 \mathrm{~cm}^{2} \mathrm{~V}^{-}$ ${ }^{1} \mathrm{~s}^{-1}$ at room temperature ${ }^{[52]}$. For p-type FET Top-gating with large-k dielectric was used with a dynamic Chanel made of a monolayer $\mathrm{WSe}_{2}$, which exhibits a mobility of $\sim 250 \mathrm{~cm}^{2} \mathrm{~V}^{-1} \mathrm{~s}^{-1}$ at room temperature, with an on/off ratio of $10^{6}$. For a top-gated $\mathrm{MoS}_{2}$ transistors with a gate length of $15 \mathrm{~nm}$ have an on/off ratio in the range of approximately $10^{10}$. Current-voltage curves for a monolayer $\mathrm{MoS}_{2}$ transistor can be described as conductivity as a function of top gate voltage for both bulk and thin-flake $\mathrm{MoS}_{2}{ }^{53]}$. Due to its high electrical performance qualities, moderately sustainable earth, abundant and good electrostatic control, could make $\mathrm{MoS}_{2}$ a suitable material for low-power electronic applications.

There are several works reported about 2D TMDC materials being used for logical transistors. By arranging numerous electrodes in different ways the monolayer $\mathrm{MoS}_{2}$ can be placed by six autonomously switchable transistors by lithographic techniques created on the same bit ${ }^{[54]}$. By the integration of two transistors with the use of monolayer $\mathrm{MoS}_{2}$, the combination worked as a logical inverter, varying its output over a consistent 0 into an intelligent 1 . Also, it can be used as universal NOR Gate, can be used for all other logical operations. Due to its high-performance mechanical properties, 2D TMDC materials can be used for the preparation of flexible devices. Mechanical studies on monolayer $\mathrm{MoS}_{2}$ states that it is 30 times as stronger compared to steel and strained up to $11 \%$ before getting the break ${ }^{[55]}$.This makes $\mathrm{MoS}_{2}$ as a good choice for making flexible transistors. Electrochemically lithiated $\mathrm{MoS}_{2}$ can be used for fabricating flexible transistors and mechanically exfoliated material can be used for sensing applications ${ }^{[56]}$. Different dichalcogenides semiconducting materials show mechanical properties as $\mathrm{MoS}_{2}$. Integration of 2D TMDCs with different $2 \mathrm{D}$ materials with conducting nature like graphene and insulating $\mathrm{BN}$ can be used for fabricating highly functionalized flexible 2D electronic devices.

With the use of molecular beam evaporation procedures, High Electron Mobility Transistors (HEMTs) can be prepared, which is another potential application of $2 \mathrm{D}$
TMDC material ${ }^{[57]}$. These devices are manufactured by the doping process, where a low band gap semiconductor is doped while a material with high band gap is left undoped, such that after the two layers are brought into contact the electrons from the doped layer transfer to the undoped layer and are allowed to move with negligible scattering from dopants. One sided Schottky contact helps for the modulation of the signal through the gate electrode. Devices that can generate measure, communicate or control the light are known as optoelectronic devices. For direct band gap semiconductor, photons with higher energy than the energy of band gap can be absorbed or emitted. While in the case of indirect band gaps, an extra phonon must be absorbed or excited to supply the distinction in momentum, making the photon assimilation or emission a great deal less proficient. Since single-layer TMDCs have essentially coordinate semiconducting band gaps, they are of extraordinary interest for applications in optoelectronics, and on the grounds that they are atomically very thin and easily applicable, they have incredible properties for adaptable and transparent optoelectronics applications.

There are diverse opportunities in subatomic sensing applications due to the electronic, optoelectronic and made wide properties of TMDCs. For containerize, intercalation here $\mathrm{Li}+$ difference at variance reasons up manners within the $\mathrm{MoS}_{2}$ Raman. The photoluminescence properties of these materials can open up the possibilities for the applications in the field of bio sensing by fluorometric tests. Later research also demonstrates that a portion of the transistors is going about as delicate locators for NO gas, which is produced using single and few-layer $\mathrm{MoS}_{2}{ }^{[58]}$. And also electrochemically tuned $\mathrm{MoS}_{2}$ material can be utilized for the detection of biomolecules ${ }^{[59]}$. Because of good mechanical properties, these materials are utilized for the flexible and transparent optoelectronics applications. Their high abundance and direct band gaps in the visible regions makes TMDCs as a suitable material for solar cell applications ${ }^{[60]}$. Compared to other materials thin films of $\mathrm{MoS}_{2}$ and $\mathrm{WS}_{2}$ are photosensitive in nature ${ }^{[61]}$. The photo responsivity and photocurrent in this device are very high. By utilizing various $\mathrm{MoS}_{2}$ layers with different thicknesses, it can be used for photograph discovery of various wavelengths. It is possible to sharpen the electrochemical solar cells of $\mathrm{TiO} 2$ with the use of $\mathrm{WS}_{2}$. TMDCs also can be utilized as conductors and to insulate layers in polymer LEDs ${ }^{[62]}$.

\section{CONCLUSION}

Transition metal dichalcogenides or TMDC materials are an important class of two-dimensional semiconducting materials with properties that enable their use in diverse areas. The unusual properties possessed by TMDCs, when reduced in size to atomically thin forms makes them attractive materials for the applications in electronics and optics. Band gap conversion from indirect to direct with decreasing size has a potential role in applications in the field of nanoelectronics. Also, the nonlinear optical properties such as SHG also show the importance of this material in optics. Compared to other 2D semiconductors 
TMDCs can be mechanically altered, which is suitable for the fabrication of flexible devices. And also the high transport properties of these materials make them a good choice for the fabrication of electronic devices. All these observations point to the idea that properties and applications of two-dimensional TDMC materials are a comparatively new, yet drastically productive and exciting area of research.

\section{REFERENCES}

[1] Novoselov, K. S. Science. 306 (5696): 666-669, (2004).

[2] Splendiani, A.; Sun, L.; Zhang, Y.; Li, T.; Kim, J.; Chim, C. Y.; Galli, G.; Wang, F. Nano Letters. 10 (4): 1271-5.(2010

[3] Mak, K. F.; Lee, C.; Hone, J.; Shan, J.; Heinz, T. F. Physical Review Letters. 105 (13) (2010).

[4] Radisavljevic, B.; Radenovic, A.; Brivio, J.; Giacometti, V.; Kis, A. Nature Nanotechnology. 6 (3): 147-50.(2011).

[5] Sundaram, R. S.; Engel, M.; Lombardo, A.; Krupke, R.; Ferrari, A. C.; Avouris, Ph; Steiner, M. Nano Letters. 13 (4): 1416-1421.(2013).

[6] Lopez-Sanchez, O.; Lembke, D.; Kayci, M.; Radenovic, A.; Kis, A. Nature Nanotechnology. 8 (7): 497-501(2013).

[7] Rycerz, A.; Tworzydło, J.; Beenakker, C. W. J. (2007). Nature Physics. 3 (3): 172.

[8] Ferrari, A. C. et al. Nanoscale7, 4598-4810 (2015).

[9] Zhu, C. R.; Wang, G.; Liu, B. L.; Marie, X.; etalPhysical Review B. 88 (12) (2013).

[10] Cao, T.; Wang, G.; Han, W.; Ye, H.; Zhu, C.; Shi, J.; etal Communications. 3 (6): 887(2012).

[11] Mak, K. F.; He, K.; Shan, J.; Heinz, T. F. Nature Nanotechnology. 7 (8): 494-8. (2012).

[12] Zeng, H.; Dai, J.; Yao, W.; Xiao, D.; Cui, X. Nature Nanotechnology. 7 (8): 490-3, (2012).

[13] Splendiani, A.; Sun, L.; Zhang etal. Nano Letters 10 (4): 1271-5 (2010).

[14] Reyes-Retana, J.A.; Cervantes-Sodi, F. Scientific Reports 6. (2016).

[15] Mak, K. F, Shan, J; Heinz, T. F. Nature Nanotechnology 7 (8): 494 (2012).

[16] Novoselov, K. S and etal, Proceedings of the National Academy of Sciences 102 (30): 10451-3. (2005).

[17] SantanuDasa , MoonkyungKimb and etal Critical Reviews in Solid State and Materials Sciences, 39:4, 231-252,

[18] Zhang, Y.; Chang, T. Rand etal Nature Nanotechnology 9 (2):. (2013)

[19] Kobayashi, K.; Yamauchi, JPhysical Review B 51 (23): 17085. (1995).

[20] Wells, A.F. Structural Inorganic Chemistry. Oxford: Clarendon Press. ISBN 0-19-855370-6 (1984).

[21] Miessler, G. L. \&Tarr, D. A. Inorganic Chemistry, 3rd Ed. Pearson/Prentice Hall publisher. ISBN 0-13035471-6. (2004).

[22] Wang, Q. H; Kalantar-Zadeh; Kis, A;etal, M.S. Nature Nanotechnology, 7, 699-712, 2007
[23] Parilla, P.; Dillon. A and etalJ. Phys. Chem. B, 108 (20), 6197-6207, 2004,

[24] Al-hilli, A.; Evans.L. Journal of Crystal Growth. 15, 93-101. 1972

[25] Tenne R, Margulis L, Genut M, Hodes G (1992).Nature 360 (6403): 444-446. 1992

[26] Eagleson, Mary, Concise encyclopedia chemistry..Retrieved 6 November 2011. (1994).

[27] Schutte, W.J.; De Boer, J.L.; Jellinek, F. Journal of Solid State Chemistry70: 207-209. (1986)

[28] Pouzet, J.; Bernede, J.C.; Khellil, A.; Essaidi, H.; Thin Solid Films 208: 252-259.1992

[29] Johnson, Dexter (11 March 2014). Retrieved 19 March 2014

[30] Castro Neto, A. H.etal. Phys. Rev. Lett. 86, 43824385 (2001).

[31] B“okerTh, Severin R, M“uller A etal Journal of Phys. Rev. B 64 235305,2001

[32] S K Mahatha, K D Patel and etal, Journal of Physics: Condensed Matter, 24, 47,2012

[33] Hua Wang, and etalNature Nanotechnology7,699712(2012)

[34] Andrea Splendiani and etal, Nanoletters, 10 (4),2010

[35] Baroni, S.; Corso, A. D.; Nano Lett. , 10 (4), pp 1271-1275,( 2010)

[36] Philipp Tonndorf, Robert Schmidt, and etal OPTICS EXPRESS Vol. 21, No. 42013

[37] S. Tongay, J. Zhou, C. Ataca, K. Lo, and etal Nano Lett. 12(11), 5576-5580 (2012).

[38] K. F. Mak, C. Lee, J. Hone, J. Shan, and T. F. Heinz, Phys. Rev. Lett. 105(13), 136805 (2010).

[39] J. A. Wilson and A. D. Yoffe, Adv. Phys. 18(73), 193-335 (1969).

[40] Weijie Zhao, ZohrehGhorannevis, , Ping-HengTan,Z and etal ACS nano, 2012

[41] M. L. Trolle, G. Seifert, and T. G. Pedersen, Phys. Rev. B 89, 235410 (2014).

[42] E. Mishina, N. Sherstyuk, and etalApplied Physics Letters 106, 131901 (2015);

[43] E. D. Mishina, N. E. Sherstyuka, and etalSemiconductors, 49, 6, 791-796(2015)

[44] C. A. Koval and J. B. Olson, J. Electroanal. Chem. 234, 133 (1987).

[45] Priya Johari and Vivek B. Shenoy ACS nano6 ,6 ' 5449-5456' 2012

[46] Wu, S. and etal.Small8, 2264-2270 (2012).

[47] Hwang, E. H., Adam, S. \& Das Sarma, S.. Phys. Rev. Lett. 98, 186806 (2007).

[48] Zhu, W., Perebeinos, V., Freitag, M. \&Avouris, P. Phys. Rev. B 80, 235402 (2009).

[49] Sakaki. H.,Noda, T., Hirakawa, K.,andetal. Appl. Phys. Lett. 51, 1934-1936 (1987).

[50] J.A. Champion; Brit. J. Appl. Phys., 16 (1965) 10351037.

[51] Podzorov. V., Gershenson, M. E. and etalAppl. Phys. Lett. 84, 3301 (2004);

[52] Jena, D. \&Konar, A. Phys. Rev. Lett. 98, 136805 (2007).

[53] Yoon. Y., Ganapathi, K. \&Salahuddin, S. Nano Lett. 11, 3768-3773 (2011). 
[54] Radisavljevic B., Whitwick, M. B. \&Kis, A. ACS Nano 5, 9934-9938 (2011).

[55] Bertolazzi.S, Brivio, J. \&Kis, A. ACS Nano 5, 97039709 (2011).

[56] He, Q. et al. Small 8, 2994-2999 (2012).

[57] Khan, M. A., Bhattarai.A, and etalAppl. Phys. Lett. 63, 1214-1215 (1993).

[58] Li, H. and etal.Small8, 63-67 (2012).

[59] Wu, S. and etal.Small8, 2264-2270 (2012).

[60] Nair, R. R. and et al. Science 320, 1308 (2008).

[61] Dean, C. R. and etal.Nature Nanotech. 5, 722-726 (2010).

[62] Friend.R. H. \&Yoffe. A. D. Adv. Phys. 36, 1-94 (1987). 